\title{
Observation of discrete energy states of weakly confined vibron in solid parahydrogen
}

\author{
$\operatorname{AUTHOR}(\mathrm{S}):$ \\ Katsuki, H; Nakamura, T; Momose, T
}

\section{CITATION:}

Katsuki, $\mathrm{H}$... [et al]. Observation of discrete energy states of weakly confined vibron in solid parahydrogen. JOURNAL OF CHEMICAL PHYSICS 2002, 116(20): 8881-8892

\section{ISSUE DATE:}

2002-05-22

URL:

http://hdl.handle.net/2433/50036

\section{RIGHT:}

Copyright 2002 American Institute of Physics. This article may be downloaded for personal use only. Any other use requires prior permission of the author and the American Institute of Physics. 


\title{
Observation of discrete energy states of weakly confined vibron in solid parahydrogen
}

\author{
Hiroyuki Katsuki, Toshihiro Nakamura, ${ }^{\text {a) }}$ and Takamasa Momose ${ }^{\text {b) }}$ \\ Department of Chemistry, Graduate School of Science, Kyoto University, and Japan Science and \\ Technology Corporation (JST), Kyoto 606-8502, Japan
}

(Received 10 December 2001; accepted 5 March 2002)

\begin{abstract}
The first overtone pure vibrational $Q_{2}(0)[v=2 \leftarrow 0, J=0 \leftarrow 0]$ transition of solid parahydrogen was studied using high-resolution laser spectroscopy. The absorption spectrum exhibits extremely narrow linewidths (20 MHz full-width-at-half-maximum), which allowed us to observe rich spectral structure in the transition. Part of the structure is attributed to the $M$ sublevel splitting of orthohydrogen that induces dipole moments on surrounding parahydrogen molecules, but the finer spectral structure is due to vibron hopping of the $v=2$ excited state of parahydrogen molecules. The Stark field of the orthohydrogen localizes the vibron on a finite number of parahydrogen molecules, which results in the discrete vibron states. From the analysis of the spectral structure, the vibron hopping frequency of the $v=2$ state is determined to be $-114 \mathrm{MHz}$. It is also found that the spectral structure cannot be explained completely unless the quantum nature of solid hydrogen is taken into account for the analysis. (C) 2002 American Institute of Physics. [DOI: 10.1063/1.1472517]
\end{abstract}

\section{INTRODUCTION}

The optical interaction of atoms and molecules in the condensed phase is intrinsically different form those in the gas phase. In the gas phase, atoms and molecules are well separated from each other so that they interact with electromagnetic radiation independently. On the other hand, due to the proximity of neighboring particles in the condensed phase compared with the wavelength of optical radiation, it happens quite often that many atoms and molecules interact with one photon simultaneously. The simultaneous excitation of multiple atoms and molecules by one photon is a characteristic feature of the optical interaction of the condensed phase.

An excited state in the condensed phase hops from one atom/molecule to others due to interatomic/molecular interactions. The eigenstate becomes delocalized, which results in a finite width of the energy state. The width of the energy corresponds to the strength of the interaction between nearest neighboring pairs, or it roughly reflects the frequency of the hopping of the excited state in the system. The energy band is filled with an infinite number of states when the excited state is delocalized over the infinite number of the constituent particles. Conversely, when the delocalization is limited to a finite number of the constituent particles, the energy state becomes discrete. Nevertheless, the spectral structure that originates in the discrete energy levels has rarely been observed spectroscopically, though many excited states in the condensed phase are considered to be partially delocalized. The reason is due to the inherent broadness of optical transitions in the condensed phase. Because of the strong inter-

\footnotetext{
${ }^{a)}$ Present address: Department of Electronic Science and Engineering, Graduate School of Engineering, Kyoto University, Kyoto 606-8502, Japan.

b) Author to whom correspondence should be addressed.
}

action and proximity between particles as well as the imperfections of solid or liquid structure, both homogeneous and inhomogeneous broadening in the condensed phase is, in general, too large to observe such spectral structure explicitly.

Solid parahydrogen, however, is an exception to this generalization. Solid hydrogen is the simplest and most fundamental molecular crystal. ${ }^{1}$ Since intermolecular interaction between hydrogen molecules is extremely weak compared with its rotational energy level spacings, each hydrogen molecule exhibits almost free rovibrational motion retaining the rotational quantum number $J$ as a good quantum number. Optical transitions to such quantized rotationvibration states have been found to exhibit extremely sharp linewidth. After the pioneering work of Hardy et al., on the high-resolution microwave absorption spectroscopy of solid parahydrogen ${ }^{2-6}$ various transitions have been studied with high-resolution. ${ }^{7-24}$ The narrowest transition so far reported is $4 \mathrm{MHz}\left(=0.0001 \mathrm{~cm}^{-1}\right)$ in the case of a pure vibrational $Q_{1}(0)$ transition $[v=1 \leftarrow 0, J=0 \leftarrow 0]$ of a $\mathrm{D}_{2}$ molecule in solid parahydrogen. ${ }^{7}$ The sharpness is a result of the small mass of hydrogen and weak intermolecular interaction. At the temperature of liquid $\mathrm{He}$, all parahydrogen molecules occupy the $J=0$ rotational state. Due to the spherical nature of the $J=0$ rotational wavefunction, the homogeneous broadening of solid parahydrogen decreases drastically. Moreover, the inhomogeneous broadening is almost negligible in solid parahydrogen because the crystal defects could be selfrepaired due to the large zero-point lattice vibration as a result of the small mass of hydrogen. Thus, both homogeneous and inhomogeneous broadening are almost completely suppressed in solid parahydrogen to exhibit extremely narrow linewidth. The narrow spectral linewidth allows us to observe fine spectral structures originating in subtle interactions in the condensed phase. 
Here we report the first high-resolution study of the pure vibrational overtone transition $Q_{2}(0)[v=2 \leftarrow 0, J=0 \leftarrow 0]$ of solid parahydrogen. The $Q_{n}(0)$ transitions in solid hydrogen become infrared active only upon interaction with internal or external electric field as is briefly described in the next section. Thus, high-resolution spectroscopy of the $Q_{n}(0)$ transitions gives us detailed and accurate information on intermolecular interactions and the nature of excited states in the condensed phase. The sharpness of the $Q_{2}(0)$ transition allowed us to directly observe discrete energy levels of the weakly confined vibron of the $v=2$ state in solid parahydrogen. Moreover, it is found that the quantum nature of solid parahydrogen can be clearly drawn from the frequency analysis of the observed spectral structure.

The mechanism of the pure vibrational transition of solid parahydrogen is briefly summarized in Sec. II. The experimental setups for high-resolution laser spectroscopy in the near-infrared region is described in Sec. III. The experimental results are given in Sec. IV, which follows the theoretical background and analysis in Secs. V and VI. The discussion on the determined parameters is given in Sec. VII.

\section{II. $Q_{n}(0)$ OPTICAL ABSORPTION IN SOLID PARAHYDROGEN}

Since the hydrogen molecule is a homonuclear diatomic molecule, the dipole transition of vibrational transition is infrared inactive. Nonetheless, various infrared absorption has been observed in solid hydrogen. Those transitions become infrared active upon interaction with the electric field. Because of the polarizability $\alpha$ of the hydrogen molecule, internal or external electric field $E$ induces dipole moment $\mu$ $=\alpha E$ on the hydrogen molecule, which interacts with photons.

Pure rotation and rotation-vibration transitions of $S$ $(\Delta J=2),{ }^{8} U(\Delta J=4),{ }^{9} W(\Delta J=6),{ }^{9-11}$ and $Y(\Delta J=8)$ (Ref. 11) types become active through instantaneous dipole moment induced by the fluctuation of the electric field of surrounding hydrogen molecules. Pure vibrational transitions of the $Q(\Delta J=0)$ type have also been observed, ${ }^{7,12-15}$ but the mechanism is different. The instantaneous fluctuation of the electric field which induces $\Delta J>0$ transitions does not induce the $Q(\Delta J=0)$ type transition. The permanent electric field is indispensable to induce the $Q$-type transition. It has shown that the $Q$-type transition of pure parahydrogen crystal is induced by external electric fields such as a laser field (stimulated Raman effect) ${ }^{16}$ or a static electric field (Condon effect). ${ }^{17,18}$ The electric field of ions produced in solid hydrogen also induces the $Q$-type transition. ${ }^{19,20}$ The observed $Q_{1}(0)$ transition induced by external fields showed extremely sharp line due to the $\Delta \mathbf{k}=0$ momentum selection rule.

On the other hand, the internal electric field of the quadrupole moment of the orthohydrogen impurity also induces the $Q$-type absorption of parahydrogen crystal. But the transition mechanism is completely different from that induced by the external electric field. Parahydrogen, having $I=0$ nuclear spin angular momentum, occupies the $J=0$ rotational state at liquid He temperature; therefore, it has no permanent multipole moment of any order. Contrarily, the orthohydrogen having the $I=1$ nuclear spin angular momentum occu- pies the $J=1$ rotational state at the low temperatures. Because of the nonspherical nature of the $J=1$ rotational wave function, the orthohydrogen has quadrupole moment, which induces dipole moment on the surrounding hydrogen molecules to cause the $Q(\Delta J=0)$ type transitions. Upon the $Q$ transitions, the inducer orthohydrogen changes its value of the quantum number $M$ which is the projection of the rotational angular momentum. Thus the pure vibrational $Q_{n}(0)$ transition induced by orthohydrogen is a simultaneous transition which should be written as $Q_{n}(0)+Q_{0}(1) .{ }^{25}$ But herein it will be referred to simply as the $Q_{n}(0)$ transition.

Since the $Q_{n}(0)$ absorption is a simultaneous transition which occurs near orthohydrogen impurities, the final state of the transition is not the same as that of the $Q_{n}(0)$ transition induced by the external field. For example, although the $v=1$ vibrational excited state of solid parahydrogen has a band width of $4 \mathrm{~cm}^{-1}$, the $Q_{1}(0)$ transition induced by external electric fields (stimulated Raman or Condon effects) shows extremely sharp linewidth, because only the $\mathbf{k}=0$ of the vibron band is optically accessible from the ground state. On the other hand, the $Q_{1}(0)$ infrared absorption of solid parahydrogen induced by orthohydrogen shows a broad spectrum with a width of $4 \mathrm{~cm}^{-1}$. Since it is a simultaneous transition with orthohydrogen, the optical selection rule $\Delta \mathbf{k}=0$ does not hold so that the entire vibron band becomes infrared active. The spectral shape is roughly proportional to the density of states of the $v=1$ vibron state, which means that the vibron is delocalized almost completely throughout the entire crystal. $^{26}$

The second overtone $Q_{3}(0)$ absorption of solid parahydrogen has been studied with high-resolution by Dickson et al. ${ }^{14,27}$ They observed several lines at approximately $11758 \mathrm{~cm}^{-1}$. The spectral structure has been interpreted as the $M$ sublevel splitting of the inducer orthohydrogen. In this case, the $v=3$ excited state is considered to be localized on a hydrogen molecule next to orthohydrogen. The breakdown of the symmetry upon excitation lifts the degeneracy of the $M$ sublevel, which was detected as a spectral structure of the $Q_{3}(0)$ absorption.

The first overtone $Q_{2}(0)$ absorption at $8070 \mathrm{~cm}^{-1}$ has been observed with low-resolution. ${ }^{28}$ A Fourier-transform infrared (FTIR) spectrum observed by Weliky et al. ${ }^{21}$ showed a doublet with a spacing of $0.5 \mathrm{~cm}^{-1}$, but no further spectral structure was resolved. Later, Mengel et al. observed the $Q_{2}(0)$ absorption at a resolution of $0.009 \mathrm{~cm}^{-1} \cdot{ }^{22}$ At low orthohydrogen concentration, they saw only the doublet reported by Weliky et al., but also a somewhat broader feature attributable, according to the analysis of Dickson et al. for $Q_{3}(0)$, to transition induced by next nearest neighbor orthohydrogen monomers. The $Q_{2}(0)$ absorption of crystals containing higher orthohydrogen concentration has also been studied by Mengel et al. They observed quite complicated spectra, which were interpreted as to the transitions of dimer, trimer and larger clusters of orthohydrogen molecules in parahydrogen crystals.

No studies of the $Q_{2}(0)$ transition with high-resolution better than $0.009 \mathrm{~cm}^{-1}$ have been performed until now because of the lack of commercially available laser sources in this frequency region. We have developed a new laser system 


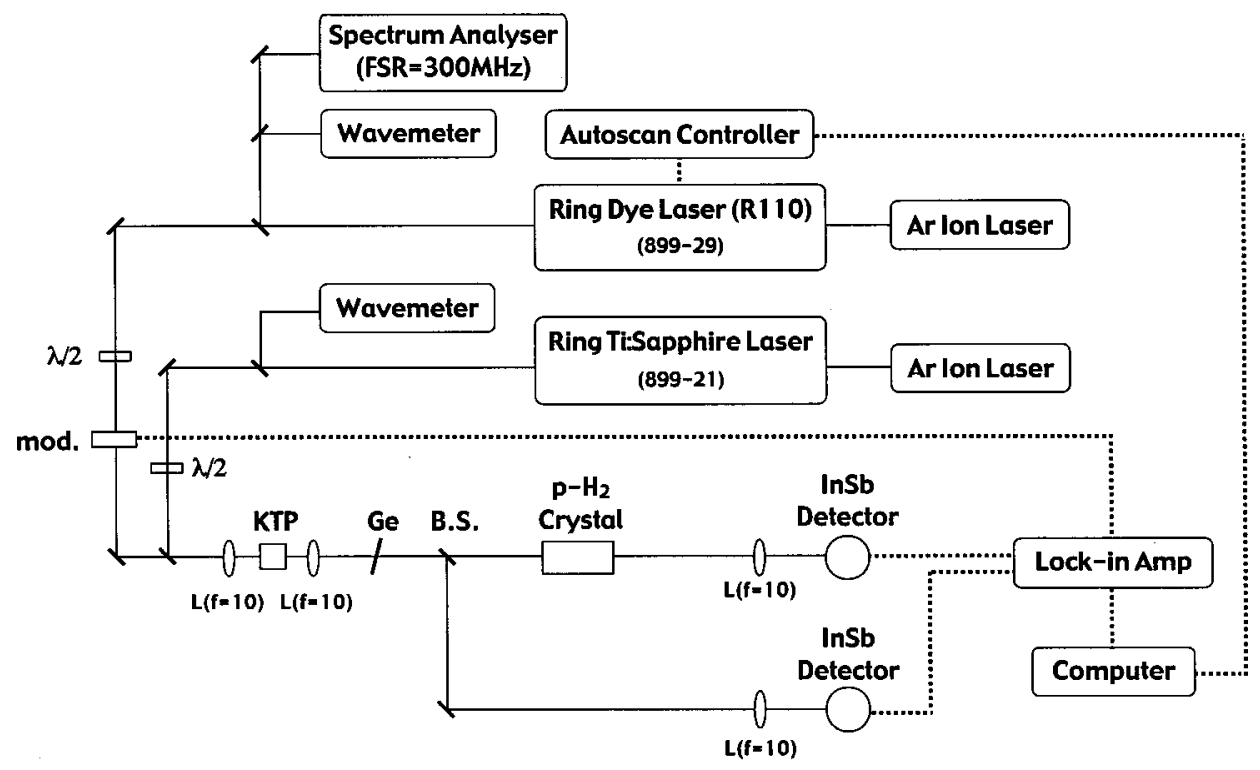

FIG. 1. The schematic diagram of the difference-frequency laser system used in this study: KTP, a potassium titanyl phosphate $\left(\mathrm{KTiOPO}_{4}\right)$ crystal; $\lambda / 2$, half-wave plate; $\mathrm{Ge}$, Germanium plate to separate IR radiation from visible; B. S., beamsplitter; $L(f=10), 10-\mathrm{cm}$ focal-length $\mathrm{BaF}_{2}$ lenses; mod, modulators (a chopper or an EO modulator for the tone-burst detection). See text for other notations. for high-resolution spectroscopy in this near infrared region. ${ }^{29}$ Here, we report our new observations of the $Q_{2}(0)$ transition of a crystal of low orthohydrogen concentration using this new laser system. We have observed more complicated spectral structure than the $Q_{3}(0)$ transition observed by Dickson et al. We found that this spectral structure is due to the discrete vibron energy levels of the $v=2$ vibron state in addition to the $M$ sublevel splitting of the inducer orthohydrogen. Details of the observed spectrum and its analysis are discussed in the following sections.

\section{EXPERIMENTS}

\section{A. Sample}

Pure parahydrogen gas was produced by passing normal liquid hydrogen through globules of hydrous ferric oxide (Ionex Research Co., Mesh size $30 \times 50$ ) kept at $13.8 \mathrm{~K}$ in a homemade ortho-para converter. ${ }^{30}$ The equilibrium orthopara ratio at this temperature was calculated to be $\sim 10^{-5}$. The parahydrogen gas thus produced was stored in a gas cylinder at room temperature and then used for the preparation of the sample. Back conversion from parahydrogen to orthohydrogen was found to be almost negligible for a few days even at room temperature, in the absence of a magnetic field.

Parahydrogen crystals were grown in an optical cell made of copper, which was attached beneath the liquid $\mathrm{He}$ reservoir of a helium cryostat. The cell was an enclosed type, and both ends were sealed with $\mathrm{BaF}_{2}$ windows for optical observations. The length and diameter of the cell were $10 \mathrm{~cm}$ and $2 \mathrm{~cm}$, respectively. Parahydrogen gas of about 100 $\mathrm{cm}^{3} / \mathrm{min}$ was introduced into the cell through a $1 / 16$ in. stainless steel tube. The cell was kept at $8.0 \mathrm{~K}$ during crystal growth. The crystal grew radially inwards from the copper surface. The entire cell except a small portion of the cylindrical center was filled with completely transparent crystal. After crystal growth, the temperature of the crystal was carefully lowered to $5.0 \mathrm{~K}$ for optical absorption experiments. At this temperature all parahydrogen molecules occupied the
$J=0$ rotational state, while orthohydrogen occupied the $J=1$ state. The actual ratio of ortho/para in this experiment was estimated to be about $1.0 \times 10^{-4}$ from the intensity of the $Q_{1}$ (1) transitions observed by a FTIR spectrometer. ${ }^{25}$ The crystal structure thus grown has been proved to be hexagonal close-packed crystal with its $c$-axis pointing towards the radial direction of the cylindrical cell. ${ }^{31}$

\section{B. Laser system}

In order to carry out high-resolution spectroscopy of the $Q_{2}(0)$ transition of solid parahydrogen at $8070 \mathrm{~cm}^{-1}$, we developed a new tunable continuous-wave (CW) midinfrared laser system based on the difference-frequency mixing. Details of the laser system have been published elsewhere. ${ }^{29}$ The block diagram of the laser system is shown in Fig. 1. Radiation from a CW ring-dye-laser (Coherent Inc., Model 899-29) at around $18000 \mathrm{~cm}^{-1}$ was mixed with another laser radiation from a $\mathrm{CW}$ ring-Ti:sapphire-laser (Coherent Inc., Model 899-21) at around $10000 \mathrm{~cm}^{-1}$ in a potassium-titanyl phosphate $\left(\mathrm{KTiOPO}_{4}\right.$; KTP) crystal to generate $\mathrm{CW}$ infrared radiation at around $8000 \mathrm{~cm}^{-1}$. The spectral purity of both ring-laser radiations was about $1 \mathrm{MHz}$. The KTP crystal, which is a biaxial crystal, was placed so that the $x y$ plane was parallel to the optical table on which all system were securely tightened. The two laser radiations were coaxially introduced at $45^{\circ}$ to the $x$ axis of the crystal in the $x y$-plane. The laser radiations were focused with a 10-cm-focal-length $\mathrm{BaF}_{2}$ lens. Configuring both input laser radiations to be horizontally polarized by half-wave plates $(\lambda / 2)$, we obtained the oscillation of difference-frequency vertically polarized relative to the optical table. The difference-frequency mixing was the type II oscillation described in the previous paper $(e-e \rightarrow o) .^{29}$ The conversion efficiency was about $5 \times 10^{-3}$; and we obtained approximately $150 \mu \mathrm{W}$ of the difference-frequency laser radiation from $200 \mathrm{~mW}$ of the dye laser and $150 \mathrm{~mW}$ of the Ti:sapphire laser radiations. The generated infrared radiation was 
separated from the two ring-laser radiations by a Ge filter, and then the radiation was introduced in a parahydrogen crystal to observe its absorption.

The frequency scan was carried out by scanning the frequencies of the dye laser radiation while fixing the frequency of the Ti:sapphire laser radiation. The scan was controlled by a computer using the AUTOSCAN program (Coherent Inc.). The frequency stability of the infrared radiation was roughly a few MHz. As for the frequency measurement, the absolute frequency of the difference-frequency radiation itself was not measured directly, but each frequency of the dye and the Ti:sapphire laser radiations was measured separately by wavemeters. Since the accuracy of the each wavemeter was $0.01 \mathrm{~cm}^{-1}$, the maximum error of the absolute wave numbers in this study could be $0.01 \mathrm{~cm}^{-1}$. However, relative wave number accuracy of the measurements was less than $0.0001 \mathrm{~cm}^{-1}$, which was ensured by the frequency stability of the two ring-laser systems. The relative wave number accuracy is important in the following discussion, but the absolute wave number is not significant.

For the detection of signals, we employed the tone-burst modulation technique ${ }^{32,33}$ in addition to the standard chopper modulation. The chopper modulation is useful for recording real spectral shape, but the tone-burst modulation is better for sensitive detection. The sensitivity $\Delta I / I$ of our tone-burst modulation is about $10^{-5}$, which is roughly two orders of magnitude better than the sensitivity of the chopper modulation. For the tone-burst modulation of this study, the side band oscillation of the dye laser radiation was generated in an EO modulator ( $\mathrm{MgO}$ doped $\mathrm{LiNbO}_{3}$ crystal) driven by radio frequencies (RF) of 5-100 $\mathrm{MHz}$. The RF was switched on and off at a rate of $30 \mathrm{kHz}$, and the signal was demodulated at this frequency by a lock-in amplifier. The tone-burst detection enhances the spectral structure whose width is close to the RF. Because of the demodulation scheme, the tone-burst spectrum appears as a second derivative-type line shape.

\section{OBSERVED SPECTRA}

Figure 2 shows a low-resolution absorption spectrum in the region of the first overtone vibrational transition. The spectrum was recorded using a Bruker FTIR spectrometer (IFS-120HR) at a resolution of $0.01 \mathrm{~cm}^{-1}$. The concentration of orthohydrogen was estimated to be less than $0.01 \%$. A sharp absorption at about $8070 \mathrm{~cm}^{-1}$ is the first overtone pure vibration transition of parahydrogen $\left[Q_{2}(0)\right]$ while the weak peak at $8060 \mathrm{~cm}^{-1}$ is that of orthohydrogen $\left[Q_{2}(1)\right]$. Broad absorption at the higher frequencies around 8150 $\mathrm{cm}^{-1}$ is the accompanying phonon band of the $Q_{2}(0)$ transition. Transitions in the region of $8300-8600 \mathrm{~cm}^{-1}$ have been assigned to the $S_{2}(0)$ transition and simultaneous transitions $Q_{1}(0)+Q_{1}(1)$ and $Q_{2}(0)+S_{0}(0)$ as annotated in Fig. $2 .^{22,34}$ With the resolution of $0.01 \mathrm{~cm}^{-1}$, the $Q_{2}(0)$ transition appeared as a doublet with a spacing of $0.3 \mathrm{~cm}^{-1}$. But it turned out that each component actually consists of many sharp lines.

The inset panel (b) of Fig. 2 shows a high-resolution spectrum of the $Q_{2}(0)$ transition observed by the differencefrequency laser system with the chopper modulation. The

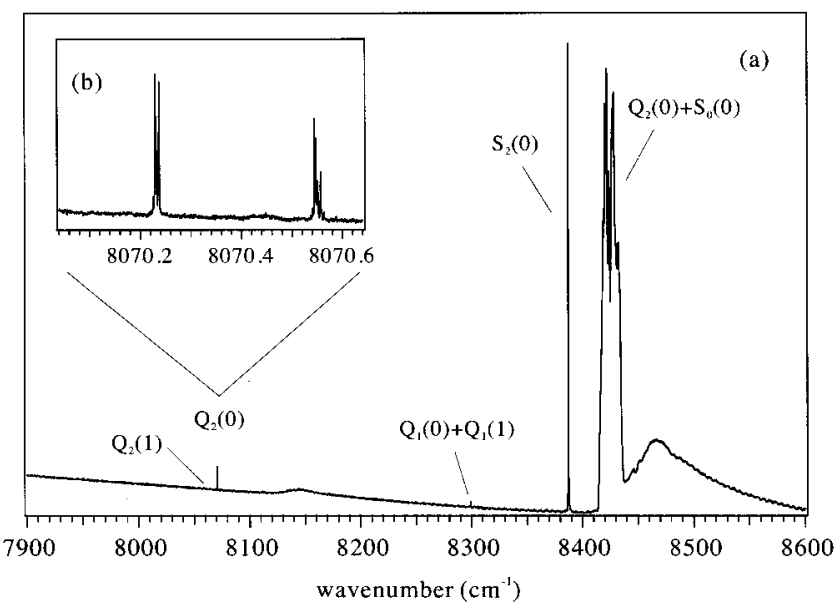

FIG. 2. Infrared absorption spectrum of solid parahydrogen in the wave number region of 7900-8600 $\mathrm{cm}^{-1}$. (a) Overview of the spectrum observed by FT spectrometer (resolution $0.01 \mathrm{~cm}^{-1}$ ). (b) High resolution spectrum of the $Q_{2}(0)$ transition observed by the difference frequency laser system with chopper modulation.

$Q_{2}(0)$ transition consists of a doublet whose lower frequency component appears at $8070.23 \mathrm{~cm}^{-1}$ and the higher component appears at $8070.55 \mathrm{~cm}^{-1}$. The spectral structure of the $Q_{2}(0)$ transition is similar to that of the $Q_{3}(0)$ previously reported, ${ }^{14}$ but the fine structure of the $Q_{2}(0)$ is more complicated than that of the $Q_{3}(0)$. The upper panel (a) of Fig. 3 shows an enlargement spectrum of each component observed at $5.0 \mathrm{~K}$, while Fig. 4 shows the spectrum observed by the tone-burst modulation with RF sideband of $10 \mathrm{MHz}$. The finer component is more clearly separated in Fig. 4 than that observed by the chopper modulation. The lower frequency component consists of 8 sharp lines, while the upper component 10 lines. The $Q_{3}(0)$ transition shows similar spectral structure with a large spacing of $0.5 \mathrm{~cm}^{-1}$, but the lower component consists of only 4 lines and the upper component, 6 lines. ${ }^{14}$ Roughly speaking, the $Q_{2}(0)$ transition has twice as many lines as the $Q_{3}(0)$ transition.

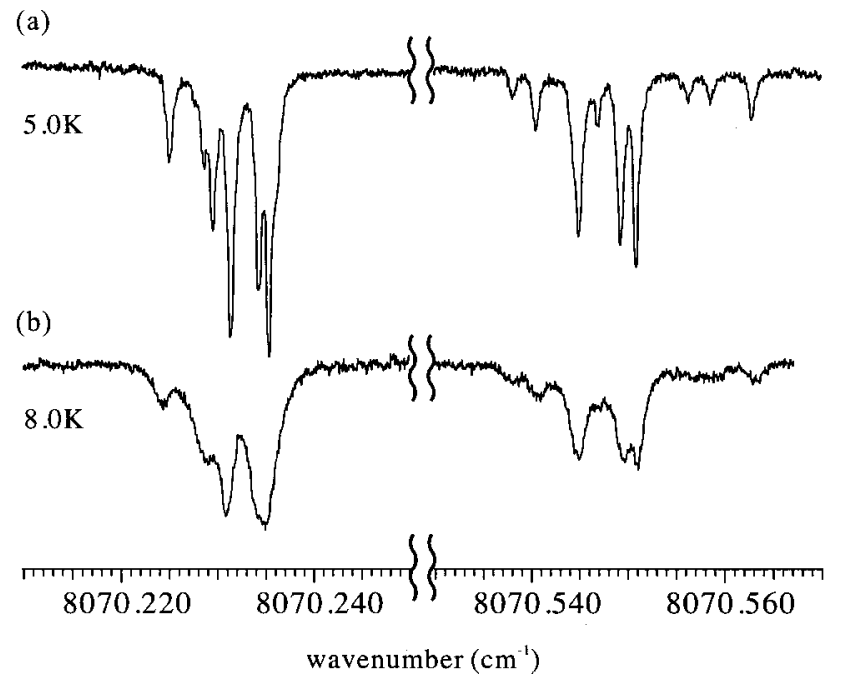

FIG. 3. Enlargement of the spectrum shown in the inset panel of Fig. 2. The chopper modulation was used. (a) The spectrum observed at $5.0 \mathrm{~K}$. (b) The spectrum observed at $8.0 \mathrm{~K}$. 

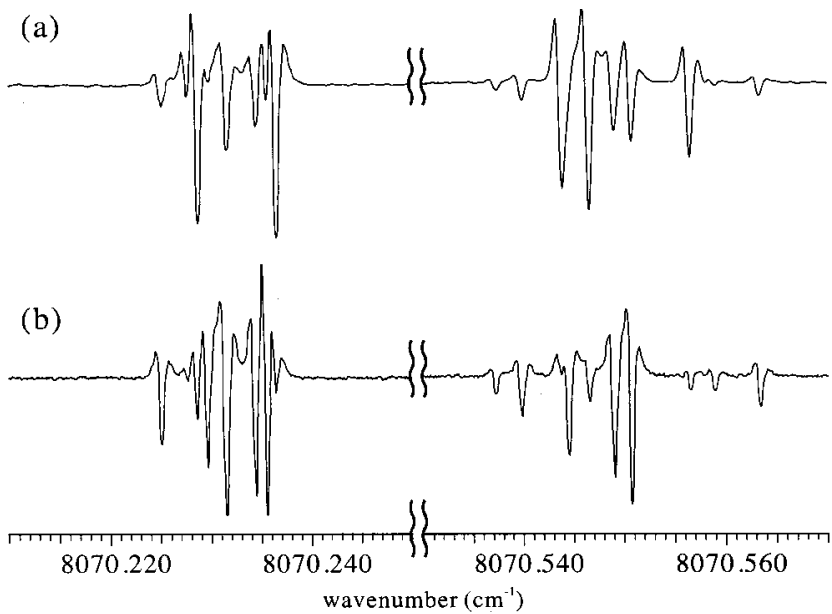

FIG. 4. High-resolution spectrum of the $Q_{2}(0)$ transition observed by the tone-burst modulation. A RF sideband of $10 \mathrm{MHz}$ was used. (a) Polarization of the laser radiation is parallel to the crystal $c$-axis. (b) Polarization is perpendicular.

The upper panel (a) of Fig. 4 shows the spectrum when the polarization of the light is parallel to the crystal $c$-axis, whereas the lower panel (b) shows the spectrum when the polarization of the light is perpendicular to the crystal $c$-axis. Each finer spectral line shows definite polarization dependence. The transition frequencies and its polarization relative to the crystal $c$-axis are summarized in Table I. As was already mentioned in the experimental section, the absolute wave number accuracy in Table I is about $0.01 \mathrm{~cm}^{-1}$, but the relative wave number accuracy among each transition is better than $0.0001 \mathrm{~cm}^{-1}$.

TABLE I. The observed and calculated frequencies of the $Q_{2}(0)$ transition induced by an isolated orthohydrogen molecule. The polarization and the assignment in terms of the extended group ${ }^{\prime} D_{3 h}^{\prime} \times{ }^{\prime} G_{4}^{\prime}$ are shown in the last two columns.

\begin{tabular}{lcccc}
\hline \hline \multicolumn{1}{c}{ Obs. } & $\begin{array}{c}\text { Wave number } \\
\left(\mathrm{cm}^{-1}\right) \\
\text { calc. }^{\mathrm{a}}\end{array}$ & $\begin{array}{c}\text { Obs. }- \text { calc. } \\
\left(\times 10^{4}\right)\end{array}$ & Polarization & $\begin{array}{c}\text { Symmetry } \\
\text { label }\end{array}$ \\
\hline Lower band & & & & \\
8070.2250 & 8070.2251 & -0.6 & perpendicular & $a E^{\prime \prime} \leftarrow a E^{\prime \prime}$ \\
8070.2276 & 8070.2277 & -1.4 & parallel & $a A_{1}^{\prime \prime} \leftarrow a A_{2}^{\prime}$ \\
8070.2287 & 8070.2285 & -1.8 & parallel & $a E^{\prime} \leftarrow a E^{\prime \prime}$ \\
8070.2297 & 8070.2296 & 1.1 & perpendicular & $a A_{1}^{\prime \prime} \leftarrow a E^{\prime \prime}$ \\
8070.2316 & 8070.2316 & -0.3 & perpendicular & $a E^{\prime \prime} \leftarrow a E^{\prime \prime}$ \\
8070.2345 & 8070.2344 & 0.7 & perpendicular & $a E^{\prime \prime} \leftarrow a E^{\prime \prime}$ \\
8070.2355 & 8070.2355 & 0.4 & perpendicular & $a E^{\prime} \leftarrow a A_{2}^{\prime}$ \\
8070.2365 & 8070.2365 & -0.3 & parallel & $a A_{1}^{\prime \prime} \leftarrow a A_{2}^{\prime}$ \\
& & & & \\
Higher band & & & perpendicular & $a E^{\prime \prime} \leftarrow a E^{\prime \prime}$ \\
8070.5371 & 8070.5371 & -0.3 & perpendicular & $a A_{1}^{\prime \prime} \leftarrow a E^{\prime \prime}$ \\
8070.5397 & 8070.5396 & 1.1 & parallel & $a E^{\prime} \leftarrow a E^{\prime \prime}$ \\
8070.5436 & 8070.5436 & -0.2 & perpendicular & $a E^{\prime \prime} \leftarrow a E^{\prime \prime}$ \\
8070.5444 & 8070.5442 & 1.9 & parallel & $a A_{1}^{\prime \prime} \leftarrow a A_{2}^{\prime}$ \\
8070.5465 & 8070.5465 & -0.3 & perpendicular & $a E^{\prime \prime} \leftarrow a E^{\prime \prime}$ \\
8070.5489 & 8070.5491 & -2.0 & perpendicular & $a E^{\prime} \leftarrow a A_{2}^{\prime}$ \\
8070.5506 & 8070.5506 & 0.4 & parallel & $a E^{\prime} \leftarrow a E^{\prime \prime}$ \\
8070.5564 & 8070.5563 & 1.2 & perpendicular & $a E^{\prime \prime} \leftarrow a E^{\prime \prime}$ \\
8070.5588 & 8070.5588 & -0.4 & perpendicular & $a E^{\prime} \leftarrow a A_{2}^{\prime}$ \\
8070.5633 & 8070.5632 & 0.8 & & \\
\hline
\end{tabular}

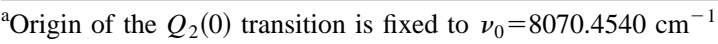

The lower panel (b) of Fig. 3 shows a chopper spectrum observed at $8.0 \mathrm{~K}$. The full-width-at-half-maximum (FWHM) of each finer spectral peak was about $20 \mathrm{MHz}$ $\left(0.0007 \mathrm{~cm}^{-1}\right)$ at $5.0 \mathrm{~K}$, but it became 1.5 times broader at $8.0 \mathrm{~K}$. Such a drastic increase of the spectral width with the rise of the temperature has been observed in the case of vibration-rotation transition of $\mathrm{CD}_{4}$ molecule embedded in parahydrogen crystals. ${ }^{35}$ The drastic increase of the width of $\mathrm{CD}_{4}$ has been interpreted to result from the pure dephasing of the vibrationally excited state. The broadening shown in Fig. 3 is also attributed to the pure dephasing of the $v=2$ vibrational excited state.

We have observed a broadening of the linewidth for crystals which contain higher concentration of orthohydrogen. This broadening is due to the inhomogeneous quadrupole field of orthohydrogen molecules in the crystal. The mechanism of the broadening has been discussed in the case of the $\mathrm{D}_{2}$ impurity transition in solid parahydrogen. ${ }^{36}$ The spectrum shown in Figs. 3 and 4 is that of the crystal containing the smallest concentration of orthohydrogen we could obtain.

Comparing these spectra with the FTIR spectra of the $Q_{2}(0)$ transition reported by Mengel et al. ${ }^{22}$ (Fig. 7 of their paper), it is obvious that the fine spectral structures shown in Figs. 3 and 4 must be attributed to the $Q_{2}(0)$ transition induced by an isolated orthohydrogen molecule in the parahydrogen crystal, but not to transitions of dimer, trimer, and higher clusters of orthohydrogen molecule.

In the case of the $Q_{3}(0)$ transition, several additional weak peaks have been observed in the middle of the large splitting of $0.5 \mathrm{~cm}^{-1}$. Dickson et al. concluded that the doublet is the $Q_{3}(0)$ transition of parahydrogen next to orthohydrogen, while the weak peaks are that of parahydrogen of next-nearest-neighbor of the orthohydrogen. We have also observed a weak transition in the middle of the doublet shown in the $Q_{2}(0)$ transition. Figure 5 shows a tone-burst spectrum of the weak transition. We observed a doublet at around $8070.45 \mathrm{~cm}^{-1}$ with a splitting of $0.0055 \mathrm{~cm}^{-1}$, but the transition was too weak to observe further spectral structure, contrary to the case of the $Q_{3}(0)$ transition.

\section{THEORETICAL FRAMEWORK}

In this section, we describe a theoretical framework for the analysis of the observed spectral structure. The general theory of rotation-vibration states of solid hydrogen has been summarized in a review ${ }^{37}$ and a book. ${ }^{1}$ A theory of the $Q_{1}(0)$ infrared absorption has been developed by Sears and Van Kranendonk. ${ }^{25}$ The theory is applied to the analysis of the $Q_{3}(0)$ absorption by Dickson et al. ${ }^{14}$ in which they have assumed that the excited state is completely localized on one hydrogen molecule. The $Q_{2}(0)$ absorption in the present study can be analyzed by the same theoretical framework, but we need to extend the theory to be able to take into account the delocalization of the vibron.

\section{A. Hamiltonian}

To make the discussion simple, we consider a pure parahydrogen crystal which contains only one orthohydrogen molecule in it. The crystal is assumed to be a hexagonal 


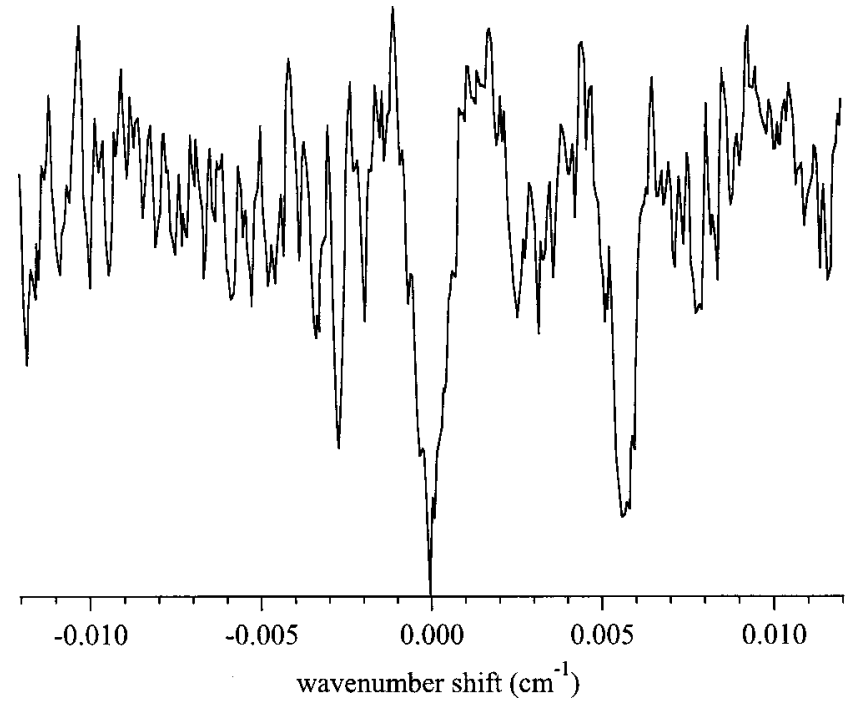

FIG. 5. Spectrum of the next-nearest-neighbor $Q_{2}(0)$ transition. The transition appears at approximately $8070.45 \mathrm{~cm}^{-1}$. The spacing of the doublet is $0.0055 \mathrm{~cm}^{-1}$. Since the absolute wave number was hard to determine with our laser system, only the wave number shift relative to the lower component of the doublet is shown.

close-packed (hcp) structure without any defects. All parahydrogen molecules occupies the $J=0$ rotational state, while the orthohydrogen occupies the $J=1$ state.

The total Hamiltonian necessary for the analysis of the $Q_{2}(0)$ transition is generally expressed as $H=H_{\text {rot-vib }}$ $+H_{\text {int }}$, where $H_{\text {rot-vib }}$ is a sum of the rotation-vibration Hamiltonian of each hydrogen molecule, and $H_{\text {int }}$ is the Hamiltonian arising from the intermolecular interaction between hydrogen molecules. We assume that the rotational quantum number $J$ is a good quantum number in the crystal; and thus, the rotation-vibration Hamiltonian is that of the standard Hamiltonian of the gas phase molecules. ${ }^{38}$ As for the interaction Hamiltonian, it can be divided into three parts as $H_{\text {int }}=V_{\text {crystal }}+H_{\text {hop }}+V_{\text {Stark }}$. The first term $V_{\text {crystal }}$ is the crystal field potential arising from the dispersion interaction between hydrogen molecules. The second term $H_{\text {hop }}$ represents the vibron hopping Hamiltonian which is responsible for the delocalization of the $v=2$ vibron in solid parahydrogen. The third term $V_{\text {Stark }}$ is the Stark field potential due to the quadrupolar field of the central orthohydrogen. Each term in $H_{\text {int }}$ is discussed separately subsequently.

\section{Crystal field potential $V_{\text {crystal }}$}

Each hydrogen molecule is under the potential which arises from the dispersion interaction between hydrogen molecules. We call this potential the "crystal field potential." In the first order approximation, the crystal field is a sum of the pair potential of every possible pair of hydrogen molecules. However, to avoid complexity, the crystal field potential of a completely pure parahydrogen crystal is taken herein as the reference of the potential. Thus, as the effective crystal field potential, we only consider the change of the pair interaction of the pure parahydrogen crystal by the substitution of an orthohydrogen molecule as well as by the vibrational excitation of the parahydrogen molecule. Since the isotropic inter- action changes only the transition frequency of the entire band, we consider only the anisotropic interaction between the orthohydrogen and parahydrogen, which changes the $M$ states of the central orthohydrogen molecules. Consequently, the isotropic interaction can be effectively included in the band origin in $H_{\text {rot-vib }}$.

The pair type interaction between orthohydrogen (molecule 1) and parahydrogen (molecule 2) is generally expressed as ${ }^{1}$

$$
A\left(R, \omega_{1}, \omega_{2}\right)=\sum_{l_{1}, l_{2}, m} A_{l_{1} l_{2} m}(R) C_{l_{1} m}\left(\omega_{1}\right) C_{l_{2} \bar{m}}\left(\omega_{2}\right),
$$

where $R$ is the intermolecular distance, and $\omega_{1}, \omega_{2}$ are the orientations of orthohydrogen and parahydrogen molecules with respect to the axis between two molecules (pair-axis). The symbol $C_{l m}(\omega)$ expresses the Racah spherical harmonics, which is related to the standard spherical harmonics $Y_{l m}(\omega)$ as

$$
C_{l m}(\omega)=\left(\frac{4 \pi}{2 l+1}\right)^{1 / 2} Y_{l m}(\omega) .
$$

The coefficient $A_{l_{1} l_{2} m}$ in Eq. (1) is a function of the distance $R$.

From the symmetry of hydrogen molecule, only even numbers of $l_{1}, l_{2}$, and $m$ survive in the summation in Eq. (1). Furthermore, since the interaction between the $J=0$ and $J=1$ states is being considered, only terms with $l_{1}=2, l_{2}=0$ have a nonzero interaction. If the distance between two hydrogen molecules are assumed to be rigidly fixed at $R_{0}$, the anisotropic interaction in Eq. (1) between the $J=0$ parahydrogen and $J=1$ orthohydrogen becomes

$$
V\left(R_{0}, \omega_{1}\right)=B_{0}\left(R_{0}\right) C_{20}\left(\omega_{1}\right),
$$

where $B_{0}\left(R_{0}\right) \equiv A_{200}\left(R_{0}\right)$. However, because of the small mass of hydrogen molecules, the amplitude of zero-point intermolecular vibration is considerably large so that the deviation from the equilibrium distance $R_{0}$ needs to be explicitly taken into account in the pair potential between hydrogen molecules. Such a treatment has been discussed by Luryi and Van Kranendonk ${ }^{39}$ as the "renormalization problem." If the instantaneous position $R$ between two molecules is considered explicitly, the anisotropic interaction potential should be written as

$$
\begin{aligned}
\widetilde{V}\left(R_{0}, \omega_{1}\right)= & \widetilde{B}_{0}\left(R_{0}\right) C_{20}\left(\omega_{1}\right) \\
& +\widetilde{B}_{2}\left(R_{0}\right)\left[C_{22}\left(\omega_{1}\right)+C_{2 \overline{2}}\left(\omega_{1}\right)\right],
\end{aligned}
$$

instead of Eq. (3). In Eq. (4), the tilde put over the potential $\widetilde{V}$ and the coefficient $\widetilde{B}_{0}, \widetilde{B}_{2}$ indicates that the instantaneous position is renormalized in them. The coefficients $\widetilde{B}_{0}$ and $\widetilde{B}_{2}$ are related to the coefficient $B_{0}$ in Eq. (3) and to the deviation of the distance, that is $\left|\mathbf{R}-\mathbf{R}_{0}\right|$, whose explicit expression is discussed in Sec. VII.

The crystal field potential of the crystal is obtained by taking all the sums of the pair interaction in Eq. (4) between the orthohydrogen and the surrounding parahydrogen molecules. Since the quantum effect discussed above is important only for the nearest-neighbor pairs but not for distant 
pairs, Eq. (4) is only applied to the pairs between orthohydrogen and its nearest-neighbor parahydrogen molecules, while Eq. (3) is used for other pairs.

The sum can be calculated by using the transformation of the coordinates from the pair-axis to the crystal-axis as

$$
C_{2 m}\left(\omega_{1}\right)=\sum_{n} D_{n m}^{2}(\mathcal{R}) C_{2 n}(\Omega),
$$

where $\Omega$ is the orientation of orthohydrogen relative to the crystal-axis and $\mathcal{R}$ is the orientation of the pair-axis between the orthohydrogen and parahydrogen with respect to the crystal axis. The function $D_{n m}^{2}(\mathcal{R})$ is the Wigner rotation matrix. ${ }^{40}$ The definition of the Wigner rotation matrix $D$ is the same as that employed by Van Kranendonk in his book. ${ }^{1}$

When all parahydrogen molecules are in the ground state, the crystal field potential is found to be

$$
V^{\mathrm{gr}}\left(\Omega, R_{0}\right)=\epsilon_{2 c} C_{20}(\Omega) .
$$

The coefficient $\epsilon_{2 c}$ is the so-called crystal field parameter. In this case, only the term proportional to $C_{20}(\Omega)$ survives because of the symmetry of the crystal around the orthohydrogen molecule, even if we use Eq. (4) for the nearest neighboring pairs.

When one of the parahydrogen molecules is in the vibrationally excited state, the crystal field potential becomes more complicated than that of the ground state. Because of the different polarizabilities for different vibrational states, pair interaction of the vibrationally excited parahydrogen with the orthohydrogen is different from that of the ground state parahydrogen. When the $j$ th parahydrogen molecule is excited to its vibrationally excited state, the crystal field potential can be expressed as

$V^{\mathrm{ex}}\left(\Omega, R_{0}\right)$

$$
\begin{aligned}
= & \epsilon_{2 c} C_{20}(\Omega)+\Delta \widetilde{B}_{0} \sum_{m^{\prime}=-2}^{2} D_{m^{\prime} 0}^{2}\left(\mathcal{R}_{j}\right) D_{m^{\prime} 0}^{2 *}(\Omega) \\
& +\Delta \widetilde{B}_{2} \sum_{m^{\prime}=-2}^{2}\left[D_{m^{\prime} 2}^{2}\left(\mathcal{R}_{j}\right)+D_{m^{\prime} \overline{2}}^{2}\left(\mathcal{R}_{j}\right)\right] D_{m^{\prime} 0}^{2 *}(\Omega),
\end{aligned}
$$

where the first term of the right-hand side is the ground state crystal field potential and the second term is the correction due to the vibrational excitation. The symbol $\mathcal{R}_{j}$ is the Euler angle which represents the orientation of the pair-axis between the $j$ th parahydrogen molecule and the orthohydrogen molecule relative to the crystal-axis. The symbol $\Delta \widetilde{B}_{n}(n=0$ or 2) represents $\widetilde{B}_{n}^{\text {ex }}-\widetilde{B}_{n}$, where $\widetilde{B}_{n}^{\text {ex }}$ is the coefficient of the pair interaction potential in Eq. (4) between the vibrationally excited parahydrogen and the orthohydrogen molecules. It should be noted that the parameter $\Delta \widetilde{B}_{2}$ related to the quantum effect appears explicitly in Eq. (7).

\section{Vibron hopping Hamiltonian $\boldsymbol{H}_{\text {hop }}$}

Delocalization of the vibrationally excited state occurs through derivatives of the dispersion interaction between molecules. Since the dispersion interaction $V_{\text {disp }}$ between two hydrogen molecules ( $i$ and $j$ ) is a function of the vibrational coordinate of each molecule, the interaction can be expanded as a power series of each vibrational coordinate $q_{i}$ and $q_{j}$ as

$$
\begin{aligned}
V_{\text {disp }}= & V_{\text {disp }}^{0}+\sum_{k=i, j} \frac{\partial V_{\text {disp }}}{\partial q_{k}} q_{k}+\sum_{k, k^{\prime}=i, j} \frac{\partial^{2} V_{\text {disp }}}{\partial q_{k} \partial q_{k^{\prime}}} q_{k} q_{k^{\prime}} \\
& +\cdots,
\end{aligned}
$$

where $V_{\text {disp }}^{0}$ is the value of $V_{\text {disp }}$ with $q_{i}=q_{j}=0$. Let $|i\rangle$ $=\left|v_{i}=2, v_{j}=0\right\rangle$ be the state in which $i$ th parahydrogen molecule is in its vibrationally excited state, and $|j\rangle=\mid v_{i}=0, v_{j}$ $=2\rangle$ be the state $j$ th molecule is in its vibrationally excited state. The two states interact with each other through the fourth derivative term in Eq. (8), because the matrix element,

$$
\sigma_{4}=\frac{\partial^{4} V_{\text {disp }}}{\partial q_{i}^{2} \partial q_{j}^{2}}\left\langle v_{i}=0, v_{j}=2\left|q_{i}^{2} q_{j}^{2}\right| v_{i}=2, v_{j}=0\right\rangle
$$

has nonzero values. Without this interaction, the two excited states degenerate. However, the nonzero matrix element in Eq. (9) lifts the degeneracy of the two states with a mixing of the wave function between $|i\rangle$ and $|j\rangle$. Physically, this interaction causes the hopping of the $v=2$ vibrational state in the crystal. The matrix element $\sigma_{4}$ in Eq. (9) for the nearest neighboring pairs is often called the hopping frequency.

The order of the hopping frequency of the pure vibrational states in solid parahydrogen has been discussed by Dickson et al. ${ }^{14}$ They showed that the hopping frequency is reduced by two orders of magnitude as the vibrational quantum number $v$ increases by one. Since the hopping frequency of the $v=1$ vibrational state is calculated to be $-5.7 \mathrm{GHz}$ $\left(=-0.19 \mathrm{~cm}^{-1}\right)$ from the spectral width of the $Q_{1}(0)$ infrared absorption, ${ }^{26,12}$ the hopping frequency of the $v=2$ vibrational state is estimated to be $-150 \mathrm{MHz}\left(=-0.005 \mathrm{~cm}^{-1}\right)$.

\section{Stark field potential $V_{\text {Stark }}$}

The electric field of the quadrupole moment of the orthohydrogen induces dipole moments on surrounding parahydrogen molecules, but it also changes the energy of surrounding hydrogen molecules by the Stark effect. It is clear that the energy shift of the 12 nearest-neighbor parahydrogen molecules to the orthohydrogen is much larger than that of the next-nearest-neighbor hydrogen molecules. The difference of the energy shift of parahydrogen molecules at different distances is of importance for the discussion of the vibron hopping, because the Stark energy appears in the diagonal matrix elements of each localized vibrationally excited state, while the vibron hopping appears in off-diagonal matrix elements.

The energy we have to consider for the analysis of vibron hopping is the difference of the Stark energy between the excited and ground vibrational state. Explicitly, the energy difference of the $i$ th parahydrogen molecule which is separated from the orthohydrogen by a distance $R_{i}$ can be written as

$$
\Delta E_{i}=-\frac{1}{2}\left(\alpha_{v=2}-\alpha_{v=0}\right) \sum_{m=-1}^{+1}(-1)^{m} \mathcal{E}_{m}\left(\mathbf{R}_{i}\right) \mathcal{E}_{-m}\left(\mathbf{R}_{i}\right),
$$


where $\mathcal{E}_{m}\left(\mathbf{R}_{i}\right)$ is the spherical component of the electric field of the quadrupole moment of the orthohydrogen at the position $\mathbf{R}_{i}$, and $\alpha_{v=2}$ and $\alpha_{v=0}$ are the polarizabilities of the parahydrogen molecule in the $v=2$ excited state and the ground state, respectively. If the energy $\Delta E_{i}$ is considerably larger compared to the hopping frequency, the vibron cannot hop from one to the other localizing the vibron in a limited region.

The electric field of the quadrupole moment of the orthohydrogen at the distance of $R=3.783 \AA$ (Ref. 41) is roughly $100 \mathrm{MV} / \mathrm{cm}$. Using the known values of $\alpha_{v=2}=6.3729$ a.u. and $\alpha_{v=0}=5.4139$ a.u., ${ }^{42}$ the $\Delta E_{i}$ at the nearest-neighbor hydrogen molecules is calculated to be $\Delta E_{n n} \sim-0.01 \mathrm{~cm}^{-1}$. The energy is about the same order as the hopping frequency given in Eq. (9) so that it is necessary to take into account for the analysis. On the other hand, the energy $\Delta E_{i}$ of the nextnearest-neighbor hydrogen molecules is calculated to be $\sim-0.0014 \mathrm{~cm}^{-1}$ which is already negligibly small.

\section{B. Basis function and its symmetry}

Rotation-vibration states in the crystal is expressed as a linear combination of the basis function which is a product of the rotation-vibration function $|J, M\rangle$ for each hydrogen molecule. We consider a crystal of $N$ molecules all in the $J=0$ rotational state and one orthohydrogen molecule in the $J=1$ rotational state. The ground state basis function is written as

$$
|M\rangle=|J=1, M\rangle \prod_{j}^{N}|v=0, J=0\rangle_{j},
$$

where $M(=0, \pm 1)$ is the projection of the rotational angular momentum $J$ of the orthohydrogen. The basis function for the vibrationally excited state in which $k$ th parahydrogen molecule is excited to the $v=2$ state is expressed as

$|M k\rangle=|J=1, M\rangle|v=2, J=0\rangle_{k} \prod_{j \neq k}^{N}|v=0, J=0\rangle_{j}$.

The eigenfunction is a linear combination of the above basis functions as

$$
|i\rangle=\sum_{M} d_{M}^{i}|M\rangle
$$

for the ground state and

$$
|j\rangle=\sum_{M k} d_{M k}^{j}|M, k\rangle
$$

for the excited state, where $d_{M}^{i}$ and $d_{M k}^{j}$ are the coefficients obtained by diagonalization of the Hamiltonian matrix described above.

The hcp lattice belongs to the $D_{3 h}$ point group, while the orthohydrogen to the $G_{4}=\left\{\bar{E}, \overline{(12)}, \bar{E}^{*}, \overline{(12)^{*}}\right\}$ permutation inversion group. Thus, by taking an appropriate linear combination, the above basis function can be symmetrized in terms of the irreducible representations of the extended group ' $D_{3 h}^{\prime} \times{ }^{\prime} G_{4}^{\prime}$. The idea of the extended group has been introduced by Reddington and Milligan for the classification of the rotation-vibration states of diatomic molecules in crystals. ${ }^{43}$ Generalization of the theory has been discussed by Miller and Decius. ${ }^{44}$ The extended group pertinent to the present system has already been discussed by Chan et al. ${ }^{24}$ This group is isomorphic to $D_{6 h}$ point group, and the character table is given in Table I of their paper.

Using the notation given in their paper, ${ }^{24}$ three ground states $(|M\rangle, M=0, \pm 1)$ are classified into $a A_{2}^{\prime}+a E^{\prime \prime}$, while the 36 vibrationally excited states for the first nearestneighbor hydrogen molecules are classified into $2 a A_{1}^{\prime}$ $+3 a A_{2}^{\prime}+4 a A_{1}^{\prime \prime}+3 a A_{2}^{\prime \prime}+5 a E^{\prime}+7 a E^{\prime \prime}$. Since the dipole moment operators transform according to $s A_{2}^{\prime \prime}+s E^{\prime}$, we expect a total of 9 parallel transitions $\left(4 a A_{1}^{\prime \prime} \leftarrow a A_{2}^{\prime}, 5 a E^{\prime}\right.$ $\left.\leftarrow a E^{\prime \prime}\right)$ and 19 perpendicular transitions $\left(5 a E^{\prime} \leftarrow a A_{2}^{\prime}\right.$, $\left.\left(4 a A_{1}^{\prime \prime}+3 a A_{2}^{\prime \prime}+7 a E^{\prime \prime}\right) \leftarrow a E^{\prime \prime}\right)$ among these states.

\section{Transition intensity}

Transition intensity is calculated from the transition dipole moment on each parahydrogen molecules induced by the electric field of quadrupole moment of orthohydrogen. Using the spherical coordinates, the electric field of quadrupole moment at a position $\mathbf{R}$ is written as ${ }^{1}$

$$
\begin{aligned}
\mathcal{E}_{m}(\mathbf{R})= & (-1)^{m} \sqrt{15} \sum_{n} Q_{2} C_{2 n}\left(\Omega_{o}\right) C(123 ; m \bar{n}) \\
& \times \frac{C_{3 n-m}^{*}(\Omega)}{R^{4}}
\end{aligned}
$$

where $Q_{2}$ is the quadrupole moment of orthohydrogen, $\Omega$ and $\Omega_{o}$ are the orientation of the vector $\mathbf{R}$ and the orthohydrogen molecule, respectively, relative to the crystal-axis, and $m=0, \pm 1$. The symbol $\mathrm{C}(123 ; m \bar{n})$ is the ClebschGordan coefficient. The $m$ th component of the induced dipole moment of a parahydrogen at $\mathbf{R}$ is expressed as

$$
\begin{aligned}
\mu_{m}= & \alpha \mathcal{E}_{m}(\mathbf{R})-\frac{\sqrt{10}}{3} \gamma \sum_{\mu} C(211 ; \mu m-\mu) \\
& \times C_{2 \mu}\left(\Omega_{p}\right) \mathcal{E}_{m-\mu}(\mathbf{R}),
\end{aligned}
$$

where $\alpha$ and $\gamma$ are the off-diagonal elements of the isotropic and anisotropic polarizabilities between the $v=0$ and $v=2$ states, respectively, $\Omega_{p}$ is the orientation of the parahydrogen molecule relative to the crystal-axis, and $m=0, \pm 1$. The second term in Eq. (16) can be disregarded in the present analysis, since we only consider pure vibrational transition of a $J=0$ molecule. Thus, the induced dipole moment on the $i$ th parahydrogen molecule becomes

$$
\begin{aligned}
\mu_{m}= & \sqrt{35} \alpha Q_{2} \frac{1}{R_{i}^{4}} \sum_{\mu} C(231 ; \mu m-\mu) C_{2 \mu}\left(\Omega_{o}\right) \\
& \times \frac{C_{3 m-\mu}^{*}\left(\Omega_{o \rightarrow i}\right)}{R_{i}^{4}},
\end{aligned}
$$

where $R_{i}$ is the distance between the $i$ th parahydrogen and the orthohydrogen molecule, and $\Omega_{o \rightarrow i}$ is the orientation of the pair-axis between the $i$ th parahydrogen and the orthohydrogen molecule relative to the crystal-axis. As a result, the 

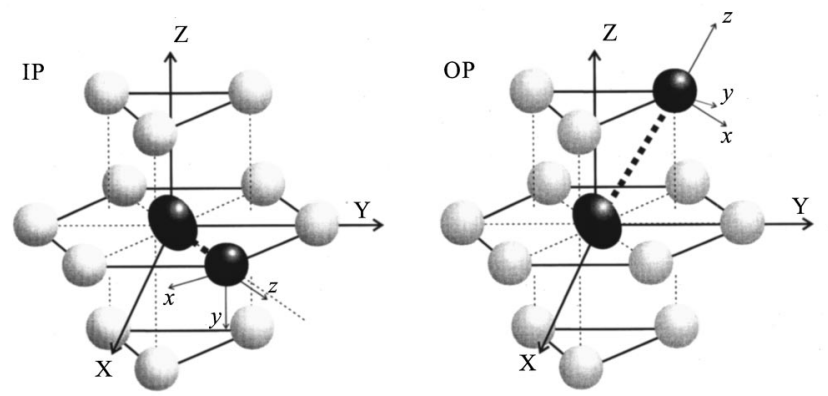

FIG. 6. In-plane (IP) and out-of-plane (OP) pair configurations. The 12 nearest-neighbors of central orthohydrogen (displayed as ellipses) are shown. Molecules in the same basal plane are connected by solid lines. The IP and OP pairs are represented by a bold broken line. The crystal-axis $(X Y Z)$ and the pair-axis $(x y z)$ are also shown.

transition moment between the ground state $|i\rangle$ in Eq. (13) and the excited state $|j\rangle$ in Eq. (14) becomes,

$$
\begin{aligned}
\left\langle j\left|\sum_{l} \mu_{m}(l)\right| i\right\rangle= & \sum_{k} \frac{\sqrt{35} Q_{2} \alpha_{20}}{R_{k}^{4}} \sum_{M M^{\prime} \mu} d_{M^{\prime} k}^{j} d_{M}^{i} \\
& \times \sum_{J} C(13 J ; M+\mu m-\mu) \\
& \times C\left(13 J ; M^{\prime} 0\right) \\
& \times D_{M+m}^{J *} m-\mu \\
& \times C(121 ; M \mu) C(231 ; \mu m-\mu),
\end{aligned}
$$

where $\alpha_{20}$ is the matrix element $\left\langle v^{\prime} J^{\prime}|\alpha| v J\right\rangle=\langle 20|\alpha| 00\rangle$. The above equation is used for the calculation of transition intensities.

\section{ANALYSIS OF THE OBSERVED SPECTRA}

Nonlinear least-squares fitting calculations were carried out to analyze the observed spectra. The parameters $\epsilon_{2 c}$, $\Delta \widetilde{B}_{0}, \Delta \widetilde{B}_{2}$, and $\sigma_{4}$ were used as the fitting parameters. The center frequency of the $Q_{2}(0)$ transition $\nu_{0}$ and the Stark stabilization energy for the nearest-neighbor parahydrogen $\Delta E_{n n}$ are fixed to the values of $\nu_{0}=8070.4540 \mathrm{~cm}^{-1}$ and $\Delta E_{n n}=-0.0109 \mathrm{~cm}^{-1}$, respectively, during the fitting procedure. As the basis function we included up to the fourth nearest-neighbor hydrogen molecules in the calculation.

Since the parameters $\Delta \widetilde{B}_{0}$ and $\Delta \widetilde{B}_{2}$ are functions of the inverse of the distance, those for next-nearest-neighbors are expected to be quite smaller than those for the nearestneighbors. Therefore, we only consider the parameters $\Delta \widetilde{B}_{0}$ and $\Delta \widetilde{B}_{2}$ for the nearest-neighbor hydrogen molecules.

There are twelve nearest-neighbor hydrogen molecules in the hcp lattice; six molecules are in the same hexagonal plane and three are in the above plane and three are in the lower plane. Thus, there are two types of nearest-neighbor hydrogen pairs in the hcp lattice, as illustrated in Fig. 6. The in-plane (IP) pair is a pair of two hydrogen molecules on the same hexagonal plane, while the out-of-plane (OP) pair is a pair in which each molecule is in a different hexagonal plane next to each other. Dickson et al. found that the $\Delta \widetilde{B}_{0}$ of IP

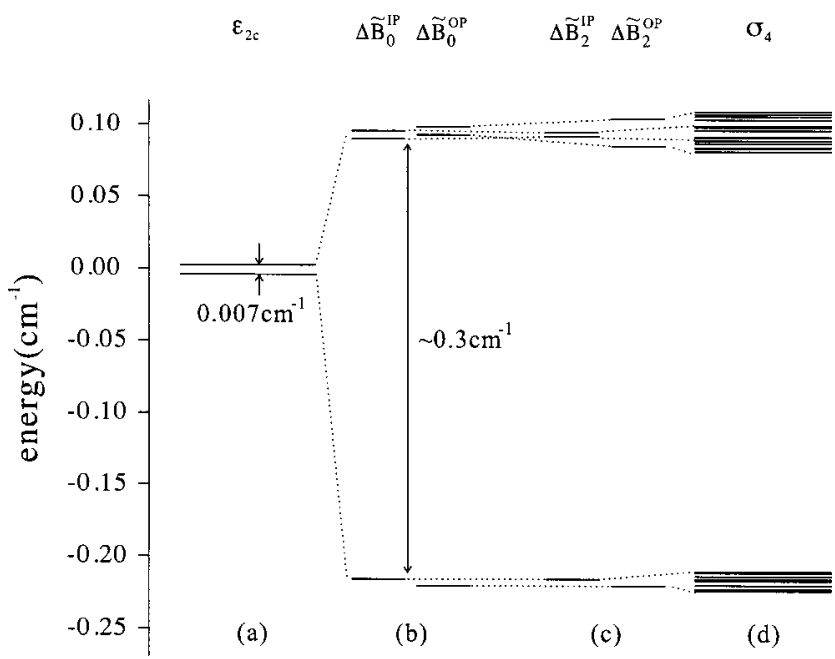

FIG. 7. Energy level diagrams of the $v=2$ vibrationally excited states. (a) Only the first term $\epsilon_{2 c}$ of Eq. (7) is considered. (b) The second term $\Delta \widetilde{B}_{0}$ is considered in addition to (a). (c) The quantum term $\Delta \widetilde{B}_{2}$ is considered in addition to (b). (d) Vibron hopping interaction is considered in addition to (c).

nearest-neighbor pairs and of OP pairs needed to be distinguished for the quantitative analysis of the $Q_{3}(0)$ spectrum. ${ }^{14}$ Since the crystal field potential of the vibrationally excited state $V^{\mathrm{ex}}(\Omega)$ is a function of the orientation of the pair-axis, the coefficients could be different between IP and OP pairs. Following Dickson's discussion, ${ }^{14}$ we also assumed that both $\Delta \widetilde{B}_{0}$ and $\Delta \widetilde{B}_{2}$ are different for the IP and OP pairs. Actually we could not obtain good fitting without using different parameters for these two coefficients. Thus, we used a total of six parameters, $\epsilon_{2 c}, \Delta \widetilde{B}_{0}^{\mathrm{IP}}, \Delta \widetilde{B}_{0}^{\mathrm{OP}}, \Delta \widetilde{B}_{2}^{\mathrm{IP}}, \Delta \widetilde{B}_{2}^{\mathrm{OP}}$, and $\sigma_{4}$ for the fitting of the spectrum.

Figure 7 depicts how the energies of the vibrationally excited states split with each interaction discussed above. The crystal field $\epsilon_{2 c}$ slightly lifts the degeneracy of $M$ into $M=0$ and $M= \pm 1$ as shown in Fig. 7(a). The crystal field potential $\Delta \widetilde{B}_{0}$ and $\Delta \widetilde{B}_{2}$ split the three $M$ levels widely-one of them has lower energy and two of them have higher energy as shown in (b) and (c) in Fig. 7. Observed large splitting of $0.3 \mathrm{~cm}^{-1}$ is due to this effect. Because of the difference of IP and OP pairs of the parameters $\Delta \widetilde{B}_{0}$ and $\Delta \widetilde{B}_{2}$, the vibrationally excited state has a total of two levels in the lower energy levels and four levels in the higher energy levels.

If we assume that the vibron is localized on one parahydrogen molecule, we expect 4 allowed transitions in the lower band and 7 transitions in the higher band. This explains the $Q_{3}(0)$ spectrum as discussed by Dickson et al. ${ }^{14}$ In the $Q_{2}(0)$ transition, however, more splitting has been observed in both lower and higher frequency bands as is seen in Figs. 3 and 4. These extra splittings are due to the vibron hopping interaction. The splitting due to the hopping is depicted in Fig. 7(d).

The energy diagram shown in Fig. 7 together with the theoretical intensities calculated from Eq. (18) allowed us to assign all the observed transitions. The parameters determined by the least squares fitting method are listed in 
TABLE II. Experimentally determined parameters. The values in parentheses are one standard deviation in the last figure.

\begin{tabular}{cc}
\hline \hline Parameter & $\mathrm{cm}^{-1}$ \\
\hline$\epsilon_{2 c}^{\mathrm{gr}}$ & $-0.0116(2)$ \\
$\Delta \widetilde{B}_{0}^{\mathrm{IP}}$ & $-0.5278(5)$ \\
$\Delta \widetilde{B}_{0}^{\mathrm{OP}}$ & $-0.5287(5)$ \\
$\Delta \widetilde{B}_{2}^{\mathrm{IP}}$ & $-0.0045(2)$ \\
$\Delta \widetilde{B}_{2}^{\mathrm{OP}}$ & $0.0149(3)$ \\
$\sigma_{4}$ & $-0.0038(1)$ \\
\hline
\end{tabular}

Table II. The comparison of the calculated stick spectrum with the experimental tone-burst spectrum are shown in Fig. 8. The calculated spectrum coincides well with the observed spectrum both in its frequency and its intensity. The calculated and observed frequencies are compared in Table I.

\section{DISCUSSION}

The ground state crystal field parameter $\epsilon_{2 c}$ is determined to be $-0.0116 \pm 0.002 \mathrm{~cm}^{-1}$. The value is slightly different from that determined previously from the analysis of the $Q_{3}(0)$ transition. ${ }^{14}$ Since our spectral resolution is much better than previous experiments, we believe that our parameter is more accurate than theirs. The higher spectral resolution is mainly due to the lower orthohydrogen concentration in our sample than that of the previous sample.

The parameter gives the crystal field splitting of

$$
\delta_{1}=E(M= \pm 1)-E(M=0)=-0.6 \epsilon_{2 c}
$$

for an orthohydrogen molecule in pure parahydrogen crystal. Determination of the value and sign of $\delta_{1}$ has been attracting much attention because it may explain the anomalous behavior of the specific heat of solid parahydrogen. ${ }^{1,45}$ The most accurate value of $\delta_{1}$ so far reported is $0.0071 \mathrm{~cm}^{-1}$ by Dick-
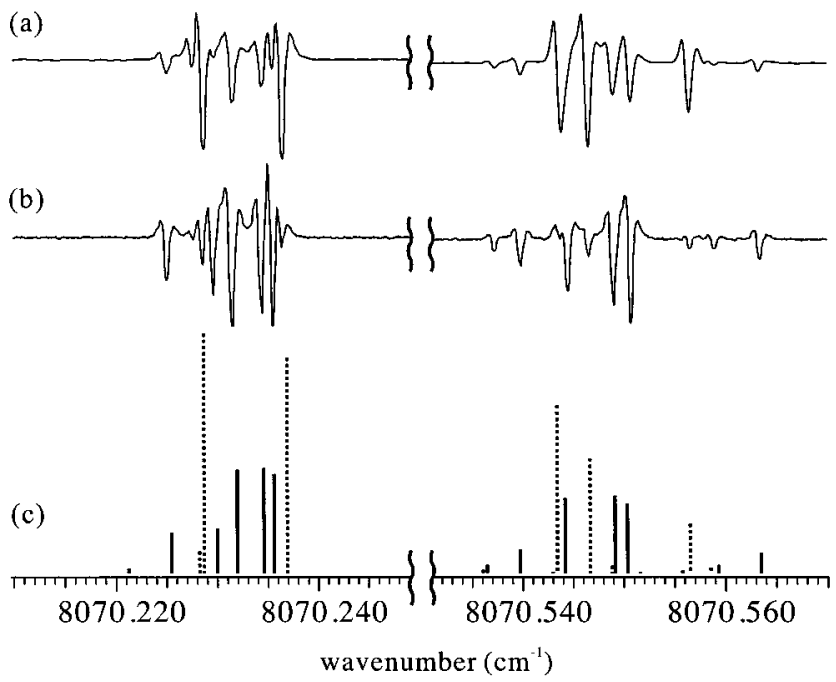

FIG. 8. Calculated stick spectrum and the observed spectra. (a) and (b) The tone-burst spectra of the $Q_{2}(0)$ transition with parallel (a) and perpendicular (b) polarizations. (c) The calculated spectrum. Transitions of perpendicular polarization are represented by solid lines, while parallel polarizations are represented by broken lines.
TABLE III. The population density distribution of the $v=2$ vibron near orthohydrogen. nn(IP): in-plane nearest-neighbor parahydrogen molecules. $\mathrm{nn}(\mathrm{OP})$ : out-of-plane nearest-neighbors. nnn: next-nearest-neighbors. 3rd $\mathrm{nn}$ : the third nearest-neighbors.

\begin{tabular}{|c|c|c|c|c|c|}
\hline $\begin{array}{l}\text { Relative } \\
\text { wave } \\
\text { numbers } \\
\left(\mathrm{cm}^{-1}\right)^{\mathrm{a}}\end{array}$ & $\begin{array}{l}\text { Sym. } \\
\text { label }\end{array}$ & $\mathrm{nn}(\mathrm{IP})$ & $\mathrm{nn}(\mathrm{OP})$ & nnn & 3rd nn \\
\hline-0.2308833 & $a A_{1}^{\prime \prime}$ & 0.2170403 & 0.7806869 & 0.0010730 & 0.0004407 \\
\hline-0.2295863 & $a A_{2}^{\prime}$ & 0.0006045 & 0.9974817 & 0.0009815 & 0.0005798 \\
\hline-0.2266261 & $a E^{\prime \prime}$ & 0.4460317 & 0.5519255 & 0.0008941 & 0.0000995 \\
\hline-0.2231692 & $a E^{\prime}$ & 0.0003541 & 0.9987197 & 0.0004525 & 0.0001515 \\
\hline-0.2220997 & $a A_{1}^{\prime \prime}$ & 0.7800766 & 0.2178437 & 0.0007153 & 0.0001405 \\
\hline-0.2200579 & $a E^{\prime \prime}$ & 0.7703447 & 0.2278295 & 0.0007283 & 0.0000240 \\
\hline-0.2172585 & $a E^{\prime \prime}$ & 0.7791056 & 0.2198541 & 0.0001788 & 0.0000303 \\
\hline-0.2161282 & $a A_{2}^{\prime \prime}$ & 0.9987696 & 0.0000902 & 0.0002771 & 0.0000000 \\
\hline 0.0827288 & $a A_{2}^{\prime}$ & 0.6116175 & 0.3686628 & 0.0101931 & 0.0007295 \\
\hline 0.0848204 & $a E^{\prime}$ & 0.3050658 & 0.6821604 & 0.0057081 & 0.0020917 \\
\hline 0.0854415 & $a E^{\prime \prime}$ & 0.1607523 & 0.8244898 & 0.0084576 & 0.0029872 \\
\hline 0.0878995 & $a A_{1}^{\prime \prime}$ & 0.0480049 & 0.9455810 & 0.0026344 & 0.0016073 \\
\hline 0.0904191 & $a A_{2}^{\prime \prime}$ & 0.8895485 & 0.0963977 & 0.0058945 & 0.0000007 \\
\hline 0.0917259 & $a A_{2}^{\prime}$ & 0.3702814 & 0.6245811 & 0.0007465 & 0.0009402 \\
\hline 0.0919333 & $a E^{\prime}$ & 0.7152671 & 0.2753844 & 0.0035373 & 0.0008212 \\
\hline 0.0925188 & $a E^{\prime \prime}$ & 0.7135718 & 0.2785926 & 0.0019853 & 0.0000002 \\
\hline 0.0968913 & $a E^{\prime \prime}$ & 0.9557367 & 0.0394052 & 0.0012980 & 0.0001265 \\
\hline 0.0974147 & $a E^{\prime}$ & 0.9090309 & 0.0842482 & 0.0023531 & 0.0002887 \\
\hline 0.0996828 & $a A_{1}^{\prime \prime}$ & 0.9479348 & 0.0473512 & 0.0015973 & 0.0000582 \\
\hline 0.0997898 & $a A_{1}^{\prime}$ & 0.9978961 & 0.0000473 & 0.0000015 & 0.0000001 \\
\hline 0.1045901 & $a E^{\prime}$ & 0.0062280 & 0.9894019 & 0.0021485 & 0.0010558 \\
\hline 0.1071567 & $a E^{\prime \prime}$ & 0.2000401 & 0.7974197 & 0.0007648 & 0.0008725 \\
\hline 0.1085640 & $a A_{1}^{\prime}$ & 0.0000000 & 0.9978726 & 0.0010460 & 0.0000000 \\
\hline 0.1103345 & $a A_{2}^{\prime \prime}$ & 0.0978302 & 0.9011295 & 0.0001968 & 0.0000000 \\
\hline
\end{tabular}

${ }^{\text {a The origin of the wave numbers is taken as the wave number of the excited }}$ state energy level without any interaction.

son et al. ${ }^{14}$ Our experimental result support their value. The $M= \pm 1$ level is above the $M=0$ level and the separation is $0.00696 \pm 0.00012 \mathrm{~cm}^{-1}=10.01 \pm 0.17 \mathrm{mK}$. More discussion on the parameter will be made in a forthcoming paper. ${ }^{46}$

The vibron hopping parameter $\sigma_{4}$ is determined to be $-0.0038 \mathrm{~cm}^{-1}$. The value is roughly the same order of magnitude as Dickson et al. predicted. In Table III, the distribution of the vibron is calculated for each excited state. The population is obtained by taking the square of the coefficients of each eigenfunction. From the table we see that about $99 \%$ of the vibron is localized on the twelve nearestneighbor parahydrogen molecules. In the case of the $v=2$ excited state, the hopping parameter is roughly on the same order of magnitude as the Stark stabilization energy. Thus, the $v=2$ vibron could distribute not only to the nearestneighbor but also to the next-nearest-neighbor and even more distant hydrogen molecules. However, the calculated result indicates that the vibron is mainly localized only to the nearest-neighbor molecules.

The localization can be explained by the effect of the crystal field parameter $\Delta \widetilde{B}_{0}$. As is depicted in Fig. $7(\mathrm{~b})$, the parameter $\Delta \widetilde{B}_{0}$ is the origin of the large splitting between the lower and upper frequency components. If we assume that the doublet shown in Fig. 5 is the transition to the vibron mainly populated on the next-nearest-neighbor or further hydrogen molecules, the splitting due to $\Delta \widetilde{B}_{0}$ for the next- 
nearest-neighbor molecules is only $0.0055 \mathrm{~cm}^{-1}$. Thus, the energy levels of the vibron on the nearest-neighbor hydrogen molecules are shifted by +0.1 or $-0.2 \mathrm{~cm}^{-1}$ due to the $\Delta \widetilde{B}_{0}$ term compared with the next-nearest-neighbor vibron. The energy difference of $+0.1 \sim-0.2 \mathrm{~cm}^{-1}$ is much larger than the hopping parameter of $-0.0038 \mathrm{~cm}^{-1}$, which suppress the hopping of the vibron from the nearest-neighbors to the next-nearest-neighbors. Comparison of the vibron hopping interaction among the $v=n(n=1,2$, and 3$)$ excited states of solid parahydrogen will be discussed in more details in a forthcoming paper. ${ }^{46}$

Next we discuss the crystal field parameters $\Delta \widetilde{B}_{0}$ and $\Delta \widetilde{B}_{2}$. For the analysis of the $Q_{3}(0)$ transition, Dickson et al. only used the parameter $\Delta \widetilde{B}_{0}$. We have tried to fit the observed spectrum by using only the parameter $\Delta \widetilde{B}_{0}^{\mathrm{IP}}$ and $\Delta \widetilde{B}_{0}^{\text {OP }}$, but we could not achieve a good fitting. We found that the inclusion of the parameters $\Delta \widetilde{B}_{2}^{\mathrm{IP}}$ and $\Delta \widetilde{B}_{2}^{\mathrm{OP}}$ drastically improve the results of fitting. The significance of the coefficients $\widetilde{B}_{0}$ and $\widetilde{B}_{2}$ in Eq. (4) has been discussed in detail by Luryi and Van Kranendonk. ${ }^{39}$ They found that the coefficients $\widetilde{B}_{0}$ and $\widetilde{B}_{2}$ are explicitly written as

$$
\begin{aligned}
\widetilde{B}_{0}(R)= & B_{0}(R)+\frac{1}{6}\left[\left\langle\mathbf{u}^{2}\right\rangle R_{0}^{-2}\left(R_{0}^{2} B_{0}^{\prime \prime}+2 R_{0} B_{0}^{\prime}-6 B_{0}\right)\right. \\
& \left.-\zeta\left(R_{0}^{2} B_{0}^{\prime \prime}-R_{0} B_{0}^{\prime}+3 B_{0}\right)\right] \\
\widetilde{B}_{2}(R)= & \frac{\sqrt{6}}{4} \eta B_{0}(R),
\end{aligned}
$$

where $B_{0}^{\prime}=\left(d B_{0}(R) / d R\right)_{R_{0}}$, and $B_{0}^{\prime \prime}=\left(d^{2} B_{0}(R) / d R^{2}\right)_{R_{0}}$. The vector $\mathbf{u}$, defined as $\mathbf{u}=\mathbf{R}-\mathbf{R}_{0}$, is the instantaneous displacement between two hydrogen molecules. The symbol $\langle\ldots\rangle$ expresses the averaging over the zero-point lattice motion. The symbols $\zeta$ and $\eta$ in Eqs. (20) and (21) represent the oblateness and nonaxiality parameters defined by

$$
\begin{aligned}
& \zeta=\frac{1}{R_{0}^{2}}\left\langle u_{x}^{2}+u_{y}^{2}-2 u_{z}^{2}\right\rangle, \\
& \eta=\frac{1}{R_{0}^{2}}\left\langle u_{x}^{2}-u_{y}^{2}\right\rangle,
\end{aligned}
$$

where $u_{x}, u_{y}$, and $u_{z}$ are the Cartesian components of the vector $\mathbf{u}$ in terms of the pair-axis shown in Fig. 6. This approximation is known as the static phonon renormalization, where the word "static" indicates that the orientation of the central orthohydrogen molecule is fixed during the averaging process.

From Eqs. (20) to (23) we see that the parameter $\widetilde{B}_{0}$ contains information on both classical and quantum effects, while the parameter $\widetilde{B}_{2}$ is a pure quantum parameter. The parameter $\widetilde{B}_{2}$ is nonzero only when the parameter $\eta$ has a finite value, which originates in the nonaxiality of the zeropoint lattice vibration. A quantitative estimation of $\eta$ in the ground state solid parahydrogen has been discussed by Hardy et al. ${ }^{2,3,5}$ and Luryi and Van Kranendonk ${ }^{39}$ based on the microwave spectrum of orthohydrogen pair transition and the neutron scattering by Nielsen. ${ }^{47}$
Luryi and Van Kranendonk discussed two models in order to estimate the quantum parameters. One is the anisotropic Debye model and the other is the perturbed Einstein model. The Debye model is based on the anisotropy of the dispersion curve of the entire phonon band. On the other hand, the Einstein model originates in the local distortion of the lattice around the pair of nearest-neighbor molecules. Since the vibron we are studying is closely localized around the impurity orthohydrogen, the perturbed Einstein model is more appropriate than the Debye model for our discussion.

It has been discussed that the nonaxiality parameter $\eta$ is different between the IP pair $\left(\eta^{\mathrm{IP}}\right)$ and the OP pair $\left(\eta^{\mathrm{OP}}\right)$. Based on the perturbed Einstein model, Luryi and Van Kranendonk have derived a relation of $\eta^{\mathrm{IP}} / \eta^{\mathrm{OP}}=-1 / 3$. From the analysis of our spectrum, we obtained the difference of the parameter $\Delta \widetilde{B}_{2}$ between the IP and OP pairs. The ratio is $\left|\Delta \widetilde{B}_{2}^{\mathrm{IP}} / \Delta \widetilde{B}_{2}^{\mathrm{OP}}\right|=0.30$. The ratio $\left|\Delta \widetilde{B}_{2}^{\mathrm{IP}} / \Delta \widetilde{B}_{2}^{\mathrm{OP}}\right|$ must be related to the ratio of $\eta^{\mathrm{IP}} / \eta^{\mathrm{OP}}$ according to Eqs. (21) and (23). Since our ratio is not the ratio $\widetilde{B}_{2}^{\mathrm{IP}} / \widetilde{B}_{2}^{\mathrm{OP}}$ of the ground state, but the ratio of the difference of the parameter $\widetilde{B}_{2}$ between the vibrationally excited state and the ground state, our ratio of $\left|\Delta \widetilde{B}_{2}^{\mathrm{IP}} / \Delta \widetilde{B}_{2}^{\mathrm{OP}}\right|=0.30$ cannot be compared directly with the ratio of $\eta^{\mathrm{IP}} / \eta^{\mathrm{OP}}=-1 / 3$, but the similarity is worth noting.

The parameter $\Delta \widetilde{B}_{0}$ is also found to be different between IP pairs and OP pairs but the difference is very small. The parameter $\Delta \widetilde{B}_{0}$ is related to the oblateness parameter $\zeta$ and the average of the zero-point deviation $\left\langle\mathbf{u}^{2}\right\rangle$ as is seen in Eq. (20). The small difference between $\Delta \widetilde{B}_{0}^{\mathrm{IP}}$ and $\Delta \widetilde{B}_{0}^{\mathrm{OP}}$ may indicate that the differences between $\zeta^{\mathrm{IP}}$ and $\zeta^{\mathrm{OP}}$, and between $\left\langle\mathbf{u}^{2}\right\rangle^{\mathrm{IP}}$ and $\left\langle\mathbf{u}^{2}\right\rangle^{\mathrm{OP}}$ are small.

There are other small interactions which we ignored in the above analysis. The $Q_{2}(0)$ excited state interacts with the double transition state $Q_{1}(0)+Q_{1}(0)$ through the second derivative of the dispersion interaction in Eq. (8) with

$$
\sigma_{2}=\frac{\partial^{2} V_{\mathrm{disp}}}{\partial q_{i} \partial q_{j}}\left\langle v_{i}=1, v_{j}=1\left|q_{i} q_{j}\right| v_{i}=2, v_{j}=0\right\rangle .
$$

The vibron hopping frequency of this type is approximately two orders of magnitude larger than the hopping in Eq. (24). Using the hopping parameter ${ }^{12} \sigma_{2}=-0.19 \mathrm{~cm}^{-1}$ and the transition frequencies of $8070 \mathrm{~cm}^{-1}$ for the $Q_{2}(0)$ and of $8300 \mathrm{~cm}^{-1}$ for the $Q_{1}(0)+Q_{1}(0)$, the second order perturbation gives the frequency shift of about $0.00015 \mathrm{~cm}^{-1}$ for the $Q_{2}(0)$ transition by this interaction. The shift of the energy is small enough so that the mixing was disregarded in the present analysis.

\section{CONCLUSION}

Herein we reported the first high-resolution spectroscopic study of the first overtone pure vibrational transition of solid parahydrogen. The overall spectral profile is similar to that of the $Q_{3}(0)$ transition, but there exists finer structures in the $Q_{2}(0)$ spectrum. We observed a total of 18 transitions. These transitions were analyzed through the consideration of the crystal field interaction and the vibron hopping interaction. By the nonlinear least-squares fitting of the observed spectra, the vibron hopping parameter $\sigma_{4}$ was determined to 
be $-0.0038 \mathrm{~cm}^{-1}=-114 \mathrm{MHz}$. In addition to this, the crystal field parameters $\Delta \widetilde{B}_{2}$ were found to be significantly different between in-plane and out-of-plane pairs. The difference of the parameter $\Delta \widetilde{B}_{2}$ is attributed to the difference of the nonaxiality of the zero-point lattice vibration, which is a pure quantum effect of the crystal.

\section{ACKNOWLEDGMENTS}

The work described herein was supported in part by the Grant-in-Aid for Scientific Research of the Ministry of Education, Science, Culture, and Sports of Japan. H.K. acknowledges the support from JSPS Research Fellowships for Young Scientists.

${ }^{1}$ J. Van Kranendonk, Solid Hydrogen, Theory of the Properties of Solid $\mathrm{H}_{2}$, $H D$, and $D_{2}$ (Plenum, New York, 1983).

${ }^{2}$ W. N. Hardy and A. J. Berlinsky, Phys. Rev. Lett. 34, 1520 (1975).

${ }^{3}$ W. N. Hardy, A. J. Berlinsky, and A. B. Harris, Can. J. Phys. 55, 1150 (1977).

${ }^{4}$ A. B. Harris, A. J. Berlinsky, and W. N. Hardy, Can. J. Phys. 55, 1180 (1977).

${ }^{5}$ B. W. Statt, W. N. Hardy, and R. Jochemsen, Can. J. Phys. 58, 1326 (1980).

${ }^{6}$ B. W. Statt and W. N. Hardy, Can. J. Phys. 58, 1341 (1980).

${ }^{7}$ D. P. Weliky, K. E. Kerr, T. J. Byers, Y. Zhang, T. Momose, and T. Oka, J. Chem. Phys. 105, 4461 (1996).

${ }^{8}$ E. Goovaerts, X. Y. Chen, A. Bouwen, and D. Schoemaker, Phys. Rev. Lett. 57, 479 (1986)

${ }^{9}$ R. A. Steinhoff, K. V. S. R. Apparao, D. W. Ferguson, K. Narahari Rao, B. P. Winnewisser, and M. Winnewisser, Can. J. Phys. 72, 1122 (1994).

${ }^{10}$ M. Okumura, M. C. Chan, and T. Oka, Phys. Rev. Lett. 62, 32 (1989).

${ }^{11}$ R. A. Steinhoff, B. P. Winnewisser, and M. Winnewisser, Phys. Rev. Lett. 73, 2833 (1994).

${ }^{12}$ Y. Zhang, T. J. Byers, M. C. Chan, T. Momose, K. E. Kerr, D. P. Weliky, and T. Oka, Phys. Rev. B 58, 218 (1998).

${ }^{13}$ M. C. Chan, L. W. Xu, C. M. Gabrys, and T. Oka, J. Chem. Phys. 95, 9404 (1991).

${ }^{14}$ R. M. Dickson, T. Momose, T. J. Byers, and T. Oka, Phys. Rev. B 57, 941 (1998).

${ }^{15}$ R. M. Dickson, T. J. Byers, and T. Oka, J. Low Temp. Phys. 102, 241 (1996).
${ }^{16}$ T. Momose, D. P. Weliky, and T. Oka, J. Mol. Spectrosc. 153, 760 (1992).

${ }^{17}$ K. E. Kerr, T. Momose, D. P. Weliky, C. M. Gabrys, and T. Oka, Phys. Rev. Lett. 72, 3957 (1994).

${ }^{18}$ T. Oka, Low Temp. Phys. 22, 96 (1996).

${ }^{19}$ T. Momose, K. E. Kerr, D. P. Weliky, C. M. Gabrys, R. M. Dickson, and T. Oka, J. Chem. Phys. 100, 7840 (1994).

${ }^{20}$ T. Momose, C. M. Lindsay, Y. Zhang, and T. Oka, Phys. Rev. Lett. 86, 4795 (2001).

${ }^{21}$ D. P. Weliky, T. J. Byers, K. E. Kerr, T. Momose, R. M. Dickson, and T. Oka, Appl. Phys. B: Lasers Opt. 59, 265 (1994).

${ }^{22}$ M. Mengel, B. P. Winnewisser, and M. Winnewisser, Phys. Rev. B 55, 10420 (1997).

${ }^{23}$ M. Mengel, B. P. Winnewisser, and M. Winnewisser, J. Mol. Spectrosc. 188, 221 (1998).

${ }^{24}$ M. C. Chan, S. S. Lee, M. Okumura, and T. Oka, J. Chem. Phys. 95, 88 (1991).

${ }^{25}$ V. F. Sears and J. Van Kranendonk, Can. J. Phys. 42, 980 (1964).

${ }^{26}$ S. K. Bose and J. D. Poll, Can. J. Phys. 68, 159 (1990).

${ }^{27}$ R. M. Dickson and T. Oka, Phys. Rev. B 57, 950 (1998).

${ }^{28}$ A. Nucara, P. Calvani, and B. Ruzicka, Phys. Rev. B 49, 6672 (1994).

${ }^{29}$ T. Momose, T. Wakabayashi, and T. Shida, J. Opt. Soc. Am. B 13, 1706 (1996).

${ }^{30}$ T. Momose and T. Shida, Bull. Chem. Soc. Jpn. 71, 1 (1998).

${ }^{31}$ T. Momose, M. Miki, T. Wakabayashi, T. Shida, M. C. Chan, S. S. Lee, and T. Oka, J. Chem. Phys. 107, 7707 (1997).

${ }^{32}$ H. M. Pickett, Appl. Opt. 19, 2745 (1980).

${ }^{33}$ C. S. Gudeman, M. H. Begemann, J. Pfaff, and R. J. Saykally, Opt. Lett. 8, 310 (1983).

${ }^{34}$ S. Y. Lee, Ph.D. thesis, The Ohio State University, 1987.

${ }^{35}$ H. Katsuki and T. Momose, Phys. Rev. Lett. 84, 3286 (2000).

${ }^{36}$ D. P. Weliky, Ph.D. thesis, University of Chicago, 1995.

${ }^{37}$ I. F. Silvera, Rev. Mod. Phys. 52, 393 (1980).

${ }^{38}$ G. Herzberg, Molecular Spectra and Structure, Vol. I, Spectra of Diatomic Molecules (Krieger, Malabar, 1989).

${ }^{39}$ S. Luryi and J. Van Kranendonk, Can. J. Phys. 57, 136 (1979); 57, 307 (1979); 57, 933 (1979).

${ }^{40}$ M. E. Rose, Elementary Theory of Angular Momentum (Wiley, New York, 1957).

${ }^{41}$ P. C. Souers, Hydrogen Properties for Fusion Energy (University of California Press, Berkeley, 1986)

${ }^{42}$ W. Kolos and L. Wolniewicz, J. Chem. Phys. 46, 1426 (1967).

${ }^{43}$ R. L. Redington and D. E. Milligan, J. Chem. Phys. 37, 2162 (1962).

${ }^{44}$ R. E. Miller and J. C. Decius, J. Chem. Phys. 59, 4871 (1973).

${ }^{45}$ A. Eucken, Sitzungsber. K. Preuss. Akad. Wiss. 1912, 41 (1912).

${ }^{46} \mathrm{H}$. Katsuki and T. Momose (in preparation).

${ }^{47}$ M. Nielsen, Phys. Rev. B 7, 1626 (1973). 